Received Date : 23-Nov-2015

Revised Date :06-Feb-2016

Accepted Date : 29-Feb-2016

Article type : Original Article

Article category: Urological Oncology

\title{
Role of Survivin expression in predicting biochemical recurrence after radical prostatectomy : a multi-institutional study
}

Romain Mathieu ${ }^{a, b}$, Ilaria Lucca ${ }^{a, c}$, Mihai Dorin Vartolomei ${ }^{a, m}$, Aurélie MBeutcha a , Tobias Klatte ${ }^{a}$, Christian Seitz ${ }^{a}$, Pierre I. Karakiewicz ${ }^{d}$, Harun Fajkovic ${ }^{a}$, Maxine Sun ${ }^{d}$, Yair Lotan ${ }^{e}$, Francesco Montorsi ${ }^{g}$, Alberto Briganti ${ }^{g}$, Morgan Rouprêt ${ }^{h}$, Vitaly Margulis ${ }^{e}$, Michael Rink ', Malte Rieken ', Lukas Kenner ${ }^{k}$, Martin Susani ${ }^{k}$, Loidl Wolgang', Shahrokh F. Shariat ${ }^{a, e, f}$

a Department of Urology, Medical University Vienna, General Hospital, Vienna, Austria

b Department of Urology, Rennes University Hospital, Rennes, France

c Department of Urology, Centre hospitalier universitaire vaudois, Lausanne, Switzerland

d Cancer Prognostics and Health Outcomes Unit, University of Montreal Health Centre, Montreal, Canada.

e Department of Urology, University of Texas Southwestern Medical Center at Dallas, Dallas, Texas.

f Department of Urology, Weill Cornell Medical College, New York, NY, USA.

g Department of Urology, Vita-Salute San Raffaele University, Milan, Italy.

h Academic Department of Urology, La Pitié-Salpetrière Hospital, Assistance Publique-Hôpitaux de Paris, Faculté de Médecine Pierre et Marie Curie, University Paris 6, Paris, France.

This article has been accepted for publication and undergone full peer review but has not been through the copyediting, typesetting, pagination and proofreading process, which may lead to differences between this version and the Version of Record. Please cite this article as doi: $10.1111 /$ bju. 13472 
i Department of Urology, University Medical Center Hamburg-Eppendorf, Hamburg, Germany.

j Department of Urology, University Hospital Basel, Basel, Switzerland

k Department of Pathology, Medical University Vienna, Vienna, Austria

I Department of Urology, Krankenhaus der Barmherzigen Schwestern, Linz, Austria

m Department of Cell and Molecular Biology, University of Medicine and Pharmacy, Targu Mures, Romania

Key words: prostatic neoplasms; Survivin; recurrence; radical prostatectomy

Short Title: Prognostic role of Survivin in prostate cancer

Corresponding author:

Shahrokh F. Shariat

Department of Urology and Comprehensive Cancer Center

Medical University of Vienna, Vienna General Hospital

Währinger Gürtel 18-20, A-1090 Vienna, Austria

Office: +4314040026150

Fax: +4314040023320

Email:shahrokh.shariat@meduniwien.ac.at

\section{ABSTRACT}

\section{Objective}

To assess the association of Survivin expression with clinicopathological features and biochemical recurrence $(\mathrm{BCR})$ after radical prostatectomy $(\mathrm{RP})$ in a large multiinstitutional cohort. 


\section{Methods}

Survivin expression was evaluated by immunohistochemistry on a tissue microarray of RP cores from 3117 patients. Survivin expression was considered altered when at least $10 \%$ of the tumor cells stained positive. Association of altered Survivin expression with BCR was evaluated using Cox proportional hazards regression models.

\section{Results}

Survivin expression was altered in 1330 (42.6\%) patients. Altered expression was associated with higher Gleason score on RP $(p=0.001)$, extracapsular extension $(p=0.019)$, seminal vesicle invasion $(p<0.001)$ and lymph node metastases $(p=0.009)$. Median follow up was 38 months (IQR 21-66). Patients with altered Survivin had a shorter BCR free survival than those with normal expression (5-year BCR free survival estimates: $74.7 \%$ vs $79.0 \%, p=0.008)$. Altered Survivin, however did not retain its prognostic value when adjusted for the effect of established clinicopathological factors $(p=0.73)$. Subgroup analyses also showed no independent prognostic value of Survivin.

\section{Conclusions}

Survivin expression is commonly altered in RP patients. Altered Survivin expression is associated with clinicopathologic features of biologically and clinically aggressive PCa. Survivin expression was associated with BCR only in univariable analysis, limiting its value in daily clinical decision making. 


\section{Introduction:}

With an estimated 220800 new cases and 27540 deaths in 2015, prostate cancer (PCa) is the most common noncutaneous malignancy in the USA ${ }^{1}$. Radical prostatectomy (RP) remains, with radiation therapy, the standard treatment for localized PCa providing durable cancer control ${ }^{2}$. However, almost one third of the patients will experience biochemical recurrence $(\mathrm{BCR})$ after these treatments. Early identification of these patients could allow adjuvant therapy which may lower the recurrence rate ${ }^{3}$. To provide a personalized approach, risk assessment and predictive tools have been developed ${ }^{4}$. These models mostly rely on clinicopathological features and fall short of optimal performance to date. Identification of new prognostic factors that could capture an individual's tumor potential could enhance current tools.

Survivin protein, a member of the inhibitors of apoptosis family, is implicated in the regulation of cell proliferation and apoptosis. Its expression has been extensively studied in various cancers making it a promising biomarker and, increasingly, target for therapy ${ }^{5}$. For example, in urothelial carcinoma of the bladder, Survivin has been shown to predict cancer recurrence and survival after radical cystectomy ${ }^{6,7}$. To date, only two small single-center studies reported a prognostic value for Survivin in $\mathrm{PCa}^{8,9}$.

Therefore, as a part of the evaluation of any potential prognostic factor ${ }^{10}$, our objective was to determine the value of Survivin as a prognostic marker for BCR in a large multi-institutional cohort of patients treated with RP. 


\section{Patient and methods:}

\section{Patient selection and data collection}

All participating sites obtained an institutional-review-board approval for the study and provided institutional data sharing agreements prior to the initiation of the study. The initial study cohort comprised 3294 patients from eight European and North American centers with PCa treated with RP between 2000 and 2011, who had tumor specimens available. We excluded patients with preoperative PSA > $50 \mathrm{ng} / \mathrm{ml}$, missing preoperative PSA, surgical margin status, lymph node status, and RP Gleason score from the analysis: 3117 patients were considered for analysis. No patient had metastases at the time of $\mathrm{RP}$ nor received preoperative radiotherapy, hormonal treatment, or chemotherapy.

\section{Pathological evaluation and immunochemistry}

All surgical specimens were processed according to standard pathologic procedures. Genito-urinary pathologists assigned pathologic stage and grade according to the 2007 American Joint Committee on Cancer (AJCC) tumor, node, and metastasis (TNM) staging system and International Society of Urological Pathology, respectively. Lymphatic tissue removed during RP was submitted for histological examination. Lymphovascular invasion (LVI) was defined as the presence of tumor cells or tumor emboli within endothelial spaces without affecting the underlying muscular wall. Tumor cells in contact with the inked surface of prostatectomy specimen defined positive surgical margins.

Survivin immunohistochemical staining was performed on tissue microarray slides in a single laboratory. Immunostaining was done on the DAKO Autostainer (DAKO 
North America) using an anti-Survivin polyclonal rabbit antibody (Novus Biologicals) at a dilution of 1:100. We used bright-field microscopy imaging coupled with advanced color detection software (Automated Cellular Imaging System; Clarient) for staining assessment. No differentiation in the intensity or the localization of the staining was considered. Survivin expression was classified "altered" when more than $10 \%$ of the cells expressed Survivin, as previously described ${ }^{7,8}$. This $10 \%$ cutoff was chosen according to a previous study that demonstrated $10 \%$ reactivity was an indicator for PSA recurrence after surgery ${ }^{8}$.

\section{Follow-up}

Follow-up (FU) was performed according to institutional protocols in agreement with local guidelines at the time. Generally, patients were seen postoperatively quarterly for the first year, semiannually in the second year, and annually thereafter. BCR was defined as a PSA value $>0.2 \mathrm{ng} / \mathrm{ml}$ on two consecutive visits. The date of BCR was attributed to the day of the first PSA. None of the patients received radiotherapy or hormone treatment before BCR.

\section{Statistical analysis}

Association of Survivin expression with categorical variables was assessed using $x^{2}$ test. Differences in continuous variables were analyzed with Kruskal-Wallis tests. Kaplan-Meier method was used to generate BCR free survival curves. Survival curves were compared using the log-rank test. Univariable and multivariable Cox regression models addressed the association of Survivin with BCR after RP. All $p$ values were two-sided, and statistical significance was defined as a $p<0.05$. 
Statistical analyses were performed using Stata 11.0 statistical software (Stata Corp., College Station, TX, USA).

\section{Results}

Survivin expression was altered in 1330 (42.6\%) patients. Clinico-pathological characteristics of overall cohort and associations with Survivin expression are shown in Table 1. Altered Survivin expression was associated with higher Gleason score on RP $\quad(p=0.001)$, extracapsular extension $(p=0.019)$, seminal vesicle invasion $(p<0.001)$, and lymph node metastases $(p=0.009)$.

Within a median follow-up of 38 (IQR 21-66) months, 617 (19.8\%) patients experimented BCR. Patients with altered Survivin expression have worse BCR free survival than those with normal expression of Survivin $(p=0.008$, Fig.1). Table 2 summarizes uni and multivariable Cox regression analyses. In univariable analyses, preoperative PSA, pathological Gleason sum, extracapsular extension, seminal vesicle invasion, surgical margin status, lymph node metastases, and altered Survivin expression were all significantly associated with BCR. In a model that adjusted for the effects of standard pathological features, Survivin did not retain its association with BCR. In subgroup analyses, Survivin did not reach association with BCR (Table 3). 


\section{Discussion}

We assessed the association of Survivin expression with pathological features of $\mathrm{PCa}$ and its value for prognosticating $\mathrm{BCR}$ in a large and multi-institutional RP cohort.

We found that more than one third of the tumors in the studied cohort expressed Survivin. This expression was associated with established features of biologically and clinically aggressive PCa such as higher Gleason score on RP and lymph node metastasis. In various cancers, Survivin expression has been already associated with adverse pathological features ${ }^{6,11,12}$. Indeed, Survivin is known to regulate tumor cell proliferation ${ }^{13}$ and apoptosis ${ }^{5,}{ }^{14}$. Basic research has already accumulated evidence regarding the anti-apoptotic role of Survivin in PCa cell lines ${ }^{15}$. Kishi et al assessed the levels of mRNA Survivin in PCa specimens and reported that Survivin expression was associated with adverse pathological features such as higher pathological $\mathrm{T}$ stage, lymph node metastasis, vessel invasion, positive surgical margin and high Gleason score ${ }^{16}$. At the protein level, our results are in line with previous studies that reported a significant association between Survivin overexpression and high Gleason scores ${ }^{8,17}$.

This association with several adverse pathological features in PCa is of great interest but does not ensure, however, that Survivin has any prognostic value. Nevertheless, prediction of recurrence and metastases spread remains one crucial issue in PCa. In the present study, we demonstrated that Survivin expression was associated with BCR in univariable analysis. However, when adjusted for standard prognostic factors, Survivin was no longer associated with impaired outcomes. These findings are similar to those previously observed in a smaller cohort ${ }^{8}$. In another study, Zhang et al distinguished nuclear and cytoplasmic expression of 
Survivin in 68 and 65 patients, respectively ${ }^{9}$. These patients were part of the RTOG 8610 study cohort, and received either radiation therapy plus short term androgen deprivation or radiation therapy alone. Immunostainings were performed in $\mathrm{PCa}$ diagnostic needle biopsies or transurethral resections. In this study, only nuclear expression was independently associated with Cancer Specific Survival and Overall Survival. Surprisingly, nuclear expression did not predict distant metastasis however. Although the number of patients is low, this study supports the potential need for subcellular discrimination of Survivin expression, especially in PCa. Indeed, Survivin may be responsible for different functions according to its expression in the cell: promotion of cell proliferation in the nucleus and control of cell survival in the cytoplasm 18, 19 . Several studies have already reported the prognostic value of cytoplasmic-to-nuclear ratio of Survivin in breast cancer ${ }^{5}$. Nevertheless, these and our results highlight the limited value of easy and readily available immunohistochemical analysis of Survivin expression in Pca.

New methods to assess Survivin expression in malignancies have however been proposed. In breast cancer, several studies demonstrated Survivin expression in circulating tumor cells was associated with adverse clinicopathological features and may have prognostic relevance regarding recurrence 20,21 . In bladder cancer, the detection of Survivin protein and mRNA in the urine has been also proposed as a diagnostic and prognostic marker ${ }^{6,22}$. Finally, Survivin expression may also be assessed in the plasma and considered as a blood marker. Indeed, high Survivin serum level at diagnosis has been reported as a prognostic factor of poor outcomes in pancreatic and ovarian carcinomas ${ }^{23,24}$. Khan et al. already demonstrated that, compared to healthy controls, levels of Survivin expression in plasma exosomes were higher in PCa patients ${ }^{25}$. These new methods to assess Survivin expression in 
urine, plasma or circulating tumor cells are promising but warrant, however, further investigations to identify any prognostic value in PCa.

We have to mention several limitations regarding the present study. First limitation is inherent to any retrospective study. Second relates to the use of immunohistochemical techniques. These techniques may limit the reliability and reproducibility of the results since discrepancies exist regarding the antibodies, technical and scoring protocols. However, tissue micro-array, readily available staining protocols and automated scoring systems based on bright-field microscopy imaging coupled with advanced color detection software were used to limit any variation at each step of the process. Accordingly, we did not discriminate between nuclear and cytoplasmic Survivin expression. Finally, we only assess early BCR in this cohort with a short follow up. However, we assume that in PCa, early BCR should be considered a reliable endpoint associated with the risk of metastasis and cancer specific mortality ${ }^{26}$.

\section{Conclusion}

Altered Survivin expression is associated with worse clinicopathologic features in PCa patients treated with RP. However, compared to standard postoperative features, altered Survivin expression is not an independent predictor of BCR after RP. Assessment of Survivin expression by standard immunostaining thus appears of limited value in clinical practice. 


\section{Ethical standards}

This study has been approved by the appropriate ethics committee.

\section{Conflict of interest}

The authors declare that they have no conflict of interest.

\section{Acknowledgements}

R.M. is supported by the Scholarship Foundation of the Republic of Austria - OeAD and by the EUSP Scholarship - European Association of Urology.

\section{References}

1. Siegel, R. L., Miller, K. D., Jemal, A.: Cancer statistics, 2015. CA Cancer J Clin, 65: 5, 2015

2. Abdollah, F., Sun, M., Thuret, R. et al.: A competing-risks analysis of survival after alternative treatment modalities for prostate cancer patients: 1988-2006. Eur Urol, 59: 88, 2011

3. Heidenreich, A., Bastian, P. J., Bellmunt, J. et al.: EAU guidelines on prostate cancer. part 1: screening, diagnosis, and local treatment with curative intent-update 2013. Eur Urol, 65: 124, 2014

4. Lughezzani, G., Briganti, A., Karakiewicz, P. I. et al.: Predictive and prognostic models in radical prostatectomy candidates: a critical analysis of the literature. Eur Urol, 58: 687, 2010

5. Rodel, F., Sprenger, T., Kaina, B. et al.: Survivin as a prognostic/predictive marker and molecular target in cancer therapy. Curr Med Chem, 19: 3679, 2012

6. Shariat, S. F., Karakiewicz, P. I., Godoy, G. et al.: Survivin as a prognostic marker for urothelial carcinoma of the bladder: a multicenter external validation study. Clin Cancer Res, 15: 7012, 2009

7. Shariat, S. F., Ashfaq, R., Karakiewicz, P. I. et al.: Survivin expression is associated with bladder cancer presence, stage, progression, and mortality. Cancer, 109: 1106, 2007

8. Shariat, S. F., Lotan, Y., Saboorian, H. et al.: Survivin expression is associated with features of biologically aggressive prostate carcinoma. Cancer, 100: 751, 2004 
9. Zhang, M., Ho, A., Hammond, E. H. et al.: Prognostic value of survivin in locally advanced prostate cancer: study based on RTOG 8610. Int J Radiat Oncol Biol Phys, 73: 1033, 2009

10. Bensalah, K., Montorsi, F., Shariat, S. F.: Challenges of cancer biomarker profiling. Eur Urol, 52: 1601, 2007

11. Kitamura, H., Torigoe, T., Hirohashi, Y. et al.: Nuclear, but not cytoplasmic, localization of survivin as a negative prognostic factor for survival in upper urinary tract urothelial carcinoma. Virchows Arch, 462: 101, 2013

12. Huang, Y. J., Qi, W. X., He, A. N. et al.: The prognostic value of survivin expression in patients with colorectal carcinoma: a meta-analysis. Jpn J Clin Oncol, 43: 988, 2013

13. Chen, W. C., Liu, Q., Fu, J. X. et al.: Expression of survivin and its significance in colorectal cancer. World J Gastroenterol, 10: 2886, 2004

14. Ambrosini, G., Adida, C., Altieri, D. C.: A novel anti-apoptosis gene, survivin, expressed in cancer and lymphoma. Nat Med, 3: 917, 1997

15. McEleny, K. R., Watson, R. W., Coffey, R. N. et al.: Inhibitors of apoptosis proteins in prostate cancer cell lines. Prostate, 51: 133, 2002

16. Kishi, H., Igawa, M., Kikuno, N. et al.: Expression of the survivin gene in prostate cancer: correlation with clinicopathological characteristics, proliferative activity and apoptosis. J Urol, 171: 1855, 2004

17. Danilewicz, M., Stasikowska-Kanicka, O., Wagrowska-Danilewicz, M.: Augmented immunoexpression of survivin correlates with parameters of aggressiveness in prostate cancer. Pol J Pathol, 66: 44, 2015

18. Li, F.: Role of survivin and its splice variants in tumorigenesis. Br J Cancer, 92: 212, 2005

19. Fortugno, P., Wall, N. R., Giodini, A. et al.: Survivin exists in immunochemically distinct subcellular pools and is involved in spindle microtubule function. J Cell Sci, 115: 575,2002

20. Yie, S. M., Lou, B., Ye, S. R. et al.: Detection of survivin-expressing circulating cancer cells (CCCs) in peripheral blood of patients with gastric and colorectal cancer reveals high risks of relapse. Ann Surg Oncol, 15: 3073, 2008

21. Yie, S. M., Luo, B., Ye, N. Y. et al.: Detection of Survivin-expressing circulating cancer cells in the peripheral blood of breast cancer patients by a RT-PCR ELISA. Clin Exp Metastasis, 23: 279, 2006

22. Ku, J. H., Godoy, G., Amiel, G. E. et al.: Urine survivin as a diagnostic biomarker for bladder cancer: a systematic review. BJU Int, 110: 630, 2012 
23. Dong, H., Qian, D., Wang, Y. et al.: Survivin expression and serum levels in pancreatic cancer. World J Surg Oncol, 13: 189, 2015

24. Dobrzycka, B., Mackowiak-Matejczyk, B., Terlikowska, K. M. et al.: Prognostic significance of pretreatment VEGF, survivin, and Smac/DIABLO serum levels in patients with serous ovarian carcinoma. Tumour Biol, 2015

25. Khan, S., Jutzy, J. M., Valenzuela, M. M. et al.: Plasma-derived exosomal survivin, a plausible biomarker for early detection of prostate cancer. PLoS One, 7: e46737, 2012

26. Freedland, S. J., Humphreys, E. B., Mangold, L. A. et al.: Risk of prostate cancerspecific mortality following biochemical recurrence after radical prostatectomy. JAMA, 294: 433, 2005 
Table 1. Association of Survivin expression and clinicopathologic characteristics in 3117 patients treated with radical prostatectomy for prostate cancer.

\begin{tabular}{|c|c|c|c|c|}
\hline & All patients & Normal Survivin & Altered Survivin & $p$ values \\
\hline Total n (\%) & 3117 & $1789(57.4)$ & $1328(42.6)$ & \\
\hline Age & & & & 0.74 \\
\hline Median (range) & $62(58-67)$ & $62(58-67)$ & $62(58-67)$ & \\
\hline Preoperative PSA & & & & 0.85 \\
\hline Median ng/ml(range) & $7(6-10)$ & $7(6-10)$ & $7(6-11)$ & \\
\hline RP Gleason sum ( $\mathrm{n} \%$ ) & & & & 0.001 \\
\hline 6 & $1,094(35.1)$ & $679(38)$ & $415(31.2)$ & \\
\hline 7 & $1,821(58.4)$ & $1007(56.2)$ & $814(61.3)$ & \\
\hline 8 & $133(4.3)$ & $69(3.9)$ & $64(4.8)$ & \\
\hline 9 & $69(2.2)$ & $34(1.9)$ & $35(2.6)$ & \\
\hline Lymph node status $\mathrm{n}(\%)$ & & & & 0.009 \\
\hline pNO & $3,044(97.7)$ & $1758(98.3)$ & $1286(96.8)$ & \\
\hline $\mathrm{pN}+$ & $73(2.3)$ & $31(1.7)$ & $42(3.2)$ & \\
\hline Lymphovascular invasion $\mathrm{n}(\%)$ & & & & 0.29 \\
\hline no & $2,793(89.6)$ & $1612(90.1)$ & $1181(88.9)$ & \\
\hline yes & $324(10.4)$ & $177(9.9)$ & $147(11.1)$ & \\
\hline Extracapsular extension ( $\mathrm{n} \%)$ & & & & 0.019 \\
\hline no & $2,292(73.5)$ & $1344(75.1)$ & $948(71.4)$ & \\
\hline yes & $825(26.5)$ & $445(24.9)$ & $380(28.6)$ & \\
\hline Seminal vesicle invasion ( $\mathrm{n} \%$ ) & & & & $<0.001$ \\
\hline no & $2,887(92.6)$ & $1684(94.1)$ & $1203(90.6)$ & \\
\hline yes & $230(7.4)$ & $105(5.9)$ & $125(9.4)$ & \\
\hline Positive surgical margin ( $\mathrm{n} \%$ ) & & & & 0.23 \\
\hline no & $2,597(83.3)$ & $1503(84)$ & $1094(82.4)$ & \\
\hline yes & $520(16.7)$ & $286(16)$ & $234(17.6)$ & \\
\hline
\end{tabular}

RP: radical prostatectomy 
Table 2 Univariable and multivariable Cox regression analyses for prediction of biochemical recurrence in 3117 patients treated with radical prostatectomy for prostate cancer with survivin status (overall analysis)

\begin{tabular}{|l|r|c|r|r|r|r|}
\hline & \multicolumn{3}{|c|}{ Univariable } & \multicolumn{3}{c|}{ Multivariable, a } \\
\cline { 2 - 7 } & \multicolumn{1}{|c|}{$\mathrm{HR}$} & $95 \% \mathrm{Cl}$ & $\mathrm{p}$ value & HR & $95 \% \mathrm{Cl}$ & \multicolumn{1}{c|}{ p value } \\
\hline Preoperative PSA & 1.06 & $1.05-1.07$ & $<0.001$ & 1.04 & $1.03-1.05$ & $<0.001$ \\
\hline RP Gleason sum & 2.65 & $2.40-2.92$ & $<0.001$ & 1.61 & $1.42-1.82$ & $<0.001$ \\
\hline Extracapsular extension & 4.59 & $3.91-5.38$ & $<0.001$ & 2.41 & $1.99-2.91$ & $<0.001$ \\
\hline Seminal vesicle invasion & 6.48 & $5.38-7.80$ & $<0.001$ & 1.66 & $1.32-2.08$ & $<0.001$ \\
\hline Positive surgical margin & 3.21 & $2.72-3.79$ & $<0.001$ & 1.96 & $1.65-2.33$ & $<0.001$ \\
\hline Lymph node metastasis & 11.75 & $9.11-15.17$ & $<0.001$ & 2.33 & $1.71-3.18$ & $<0.001$ \\
\hline Survivin & 1.24 & $1.06-1.45$ & 0.008 & 1.03 & $0.88-1.21$ & 0.73 \\
\hline
\end{tabular}

$\mathrm{Cl}$ confidence interval, HR hazard ratio, RP radical prostatectomy

a. Multivariable Cox regression adjusted for PSA value, RP Gleason score, lymph node involvement, positive surgical margins, extracapsular extension, seminal vesicle invasion, and survivin status. 
Table 3 Multivariable Cox regression analyses for prediction of biochemical recurrence according to Survivin status in subgroups of patients treated with radical prostatectomy

\begin{tabular}{|l|c|c|c|}
\hline \multirow{2}{*}{ Patient subgroup } & \multicolumn{3}{c|}{ Multivariable } \\
\cline { 2 - 4 } & $\mathrm{HR}$ & $95 \% \mathrm{Cl}$ & $\mathrm{p}$ value \\
\hline No lymph node involvement, a & 1.05 & $0.88-1.24$ & 0.60 \\
\hline Lymph node involvement, a & 0.90 & $0.52-1.55$ & 0.71 \\
\hline Negative surgical margins, b & 1.03 & $0.84-1.27$ & 0.77 \\
\hline Positive surgical margins, b & 1.04 & $0.79-1.35$ & 0.82 \\
\hline $\begin{array}{l}\text { Locally advanced disease (pN0 and Stage } \\
\text { pT3a/b pN0), c }\end{array}$ & 1.09 & $0.88-1.35$ & 0.43 \\
\hline $\begin{array}{l}\text { Localized disease (pN0 and no Stage } \\
\text { pT3a/b pN0), c }\end{array}$ & 1.06 & $0.83-1.34$ & 0.62 \\
\hline RP Gleason sum < ou =6, d & 0.91 & $0.61-1.35$ & 0.65 \\
\hline RP Gleason sum = 7, d & 1.04 & $0.85-1.27$ & 0.72 \\
\hline RP Gleason sum > ou = 8, d & 1.20 & $0.82-1.74$ & 0.35 \\
\hline
\end{tabular}

$\mathrm{Cl}$ confidence interval, HR hazard ratio, RP radical prostatectomy

a Multivariable Cox regression adjusted for PSA value, RP Gleason score, positive surgical margins, extracapsular extension, seminal vesicle invasion.

b Multivariable Cox regression adjusted for PSA value, RP Gleason score, lymph node involvement, extracapsular extension, seminal vesicle invasion c Multivariable Cox regression adjusted for PSA value, RP Gleason score, d Multivariable Cox regression adjusted for PSA value, lymph node involvement, positive surgical margins, extracapsular extension, seminal vesicle invasion 
Figure 1. Biochemical recurrence free survival estimates according to the expression of Survivin expression in the cohort of 3117 patients treated with radical prostatectomy.

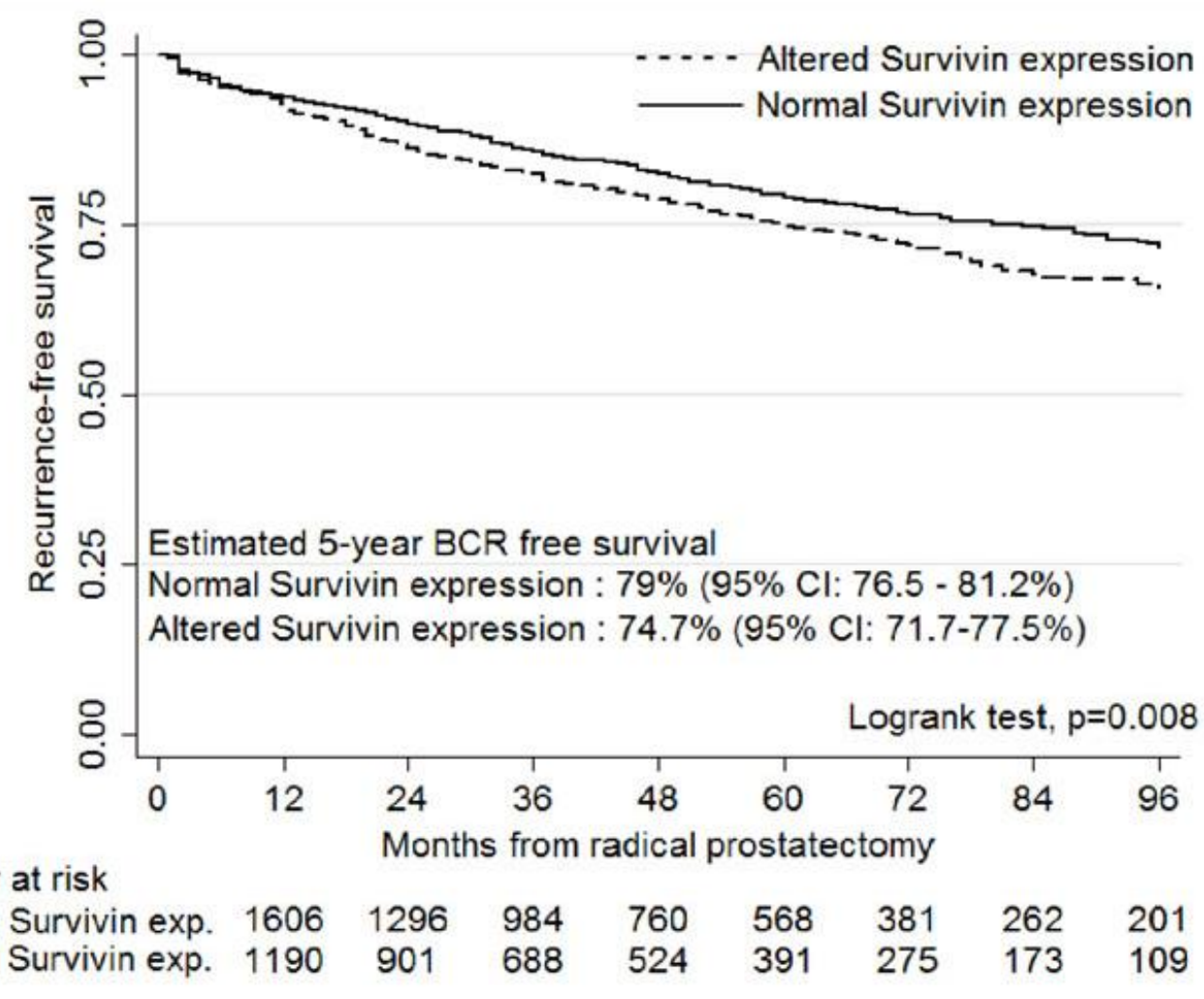

Number at risk

$\begin{array}{lllllllll}\text { Altered Survivin exp. } 1606 & 1296 & 984 & 760 & 568 & 381 & 262 & 201\end{array}$

$\begin{array}{llllllll}\text { Normal Survivin exp. } 1190 & 901 & 688 & 524 & 391 & 275 & 173 & 109\end{array}$

